\title{
Indicadores urbanos y su influencia en el desarrollo sostenible urbano de Huancayo metropolitano - Perú*
}

\author{
Urban indicators and their influence on the urban sustainable \\ development of metropolitan Huancayo - Peru
}

\author{
Cesar Fortunato Martínez Vitor ${ }^{1 *}$
}

Recibido 8 de mayo de 2017, aceptado 24 de mayo de 2018

Received: May 8, 2017 Accepted: May 24, 2018

\begin{abstract}
RESUMEN
El estudio tuvo como propósito identificar la influencia de los indicadores urbanos en el desarrollo sostenible urbano de Huancayo Metropolitano, región Junín - Perú, con sus componentes específicos de configuración de la ciudad y el territorio, gestión de los recursos naturales, cohesión social y desarrollo económico en el desarrollo sostenible urbano. Se aplicaron tres metodologías: la primera de indicadores de la cartografía evolutiva y el sistema de información censal, la segunda es la contrastación de fichas metodológicas del sistema integrado de indicadores urbanos con los planes urbanos realizados desde 1961 hasta 2011 y la tercera la metodología para estimar el nivel de desarrollo sostenible de territorios, biograma 2008 elaborado por el Instituto Interamericano de Cooperación para la Agricultura (IICA). Logrando con ellos obtener los siguientes resultados: la densidad bruta para el año 1961 de 115,87 hab/ha ha decrecido sostenidamente llegando hasta el año 2011 con 93,67 hab/ha , la contrastación de los planes urbanos realizados desde 1961 hasta el año 2011, da como resultado que de 14 indicadores obtenidos, solo 1 aplica, resaltando que 13 indicadores no aplican por fallas metodológicas, los cuales no permiten seguir el monitoreo al carecer de confiabilidad, finalmente el biograma muestra la tendencia hacia la estabilidad urbana. Los resultados muestran la utilidad práctica de los indicadores urbanos demostrando con ello el grado de influencia en la situación actual y previsible del hábitat urbano que, a pesar de tener un crecimiento vegetativo de manera inercial y espontáneo, conducen a acrecentar la sostenibilidad a mediano plazo.
\end{abstract}

Palabras clave: Ciudad metropolitana, desarrollo sostenible, indicadores urbanos, sostenibilidad urbana.

\begin{abstract}
The purpose of the research was to identify the influence of urban indicators on the urban sustainable development of metropolitan Huancayo, Junín region - Peru, including its specific city components and territory configuration, management of natural resources, social cohesion and economic development in urban sustainable development. Three methodologies were applied: the first of evolutionary mapping indicators and the census information system, the second was the testing of methodological records of the integrated system of urban indicators with the urban plans made from 1961 to 2011 and the third methodology for estimating the level of sustainable development of territories, Biogram 2008 prepared by the Inter-American Institute for Cooperation on Agriculture (IICA). Achieving the following results:

\footnotetext{
1 Universidad Nacional del Centro del Perú. E-mail: cesar_vitor@ hotmail.com

* Tesis sustentada en la Escuela Universitaria de Post Grado de la Universidad Nacional Federico Villareal - Perú, para optar el grado académico de doctor en Medio Ambiente y Desarrollo Sostenible.

* Autor de correspondencia
} 
the 1961 bulk density of $115.87 \mathrm{dpHa}$ has decreased steadily reaching in 2011 until 93, $67 \mathrm{dpHa}$, the contrasting urban plans made from 1961 to 2011, from 14 indicators obtained, only 1 applies, noting that 13 indicators do not apply due to methodological flaws, which do not allow monitoring to be followed due to lack of reliability, finally the biogram shows us the trend toward urban stability. The results show the practical usefulness of the urban indicators, thus demonstrating the degree of influence in the current and predictable situation of the urban habitat that, despite having a vegetative growth in an inertial and spontaneous way, lead to increase the sustainability in the medium term.

Keywords: Metropolitan city, sustainable development, urban Indicators, urban sustainability.

\section{INTRODUCCIÓN}

El valle del Mantaro representa un ecosistema especial en el contexto ambiental peruano, cuenta con suelos de alta capacidad productiva, microcuencas hidrográficas perennes y paisajes naturales de mucha belleza. En el estudio efectuado por Martínez [1] sobre la vulnerabilidad ambiental en valle del Mantaro, por las actividades urbanas se estableció que el crecimiento y dinámica de las ciudades conllevan a la vulnerabilidad del valle del Mantaro, incluso a desequilibrios ambientales, si se superan los límites de impacto permisibles, generándose conflictos entre la ciudad y el territorio mediante la ocupación desordenada del suelo agrícola para asentamientos urbanos; extracción descontrolada de recursos naturales; consumo excesivo del agua; contaminación del medio atmosférico, agua y suelo; perdida del paisaje y costumbres. Por ello, se vio la necesidad de interiorizar sobre las causas de este proceso, y entre ellas se encuentra que los gobernantes y la población han tomado decisiones y procesos inadecuados sin ningún sistema de control y monitoreo por ello se hace imprescindible establecer un sistema de indicadores urbanos.

Para poder insertarse en la tendencia mundial de trabajar con indicadores de gestión urbana es un reto para los países en desarrollo y más aún para las ciudades metropolitanas con un rango de población de 500,000 a 999,999 [2], como es el caso de Huancayo donde no existe ningún tipo de antecedente, y que esta les permita tener una base de datos, procesos de control estadístico, indicadores e índices para el diseño de políticas, estrategias, acciones y proyectos de desarrollo urbano.

Considerando que la gestión urbana en el sistema nacional peruano y de la ciudad de Huancayo no se basa en indicadores urbanos sistémicos, esto ocasiona inadecuadas tomas de decisiones en proyectos de desarrollo urbano. Como se puede evidenciar en la formulación de 17 planes urbanos desde 1943 hasta el plan 2006-2011, las cuales fueron rebasadas por la realidad, y no se cumplieron las metas propuestas, generando entre otros: un crecimiento urbano espontáneo, estructura urbana física espacial desarticulada del centro a la periferia, apropiación de las tierras de cultivo para los usos urbanos, sin suficiente acompañamiento de los servicios básicos y equipamiento a ello se suma que el sector de Vivienda Construcción y Saneamiento, al no implementar el observatorio urbano, y la elaboración de indicadores urbanos, se condujo a la desinformación de la población sobre los aspectos relacionados a la calidad de vida urbana.

Desde el programa 21 [3], hasta la actualidad, la construcción y aplicación de indicadores de desarrollo urbano sostenible en América Latina, se viene aplicando a países como Brasil, México, Argentina, Colombia, Chile, y otros.

Para nuestro caso se ha previsto en el decreto supremo $\mathrm{N}^{\circ}$ 022-2016-VIVIENDA elaborado por el Ministerio de Vivienda Construcción y Saneamiento [4], donde reglamentan en su artículo 77 que Los Gobiernos Locales implementan los Observatorios Urbanos Locales para la evaluación y monitoreo del PAT, del PDM, del PDU y del EU, sobre la base de los indicadores de evaluación y seguimiento, definidos en los respectivos planes, de ahí la necesidad urgente de iniciar su implementación de esta herramienta de gestión y planeamiento e integrarnos al sistema de medición de los observatorios urbanos y propender al desarrollo sostenible.

Es posible que los gobiernos locales inicien con este proceso mientras que el problema se mantiene latente. 
Los problemas descritos a través de las tres dimensiones o factores que agrupan el contexto urbano, se explican a través de los indicadores urbanos cuya situación existente se observa en la Figura 1.

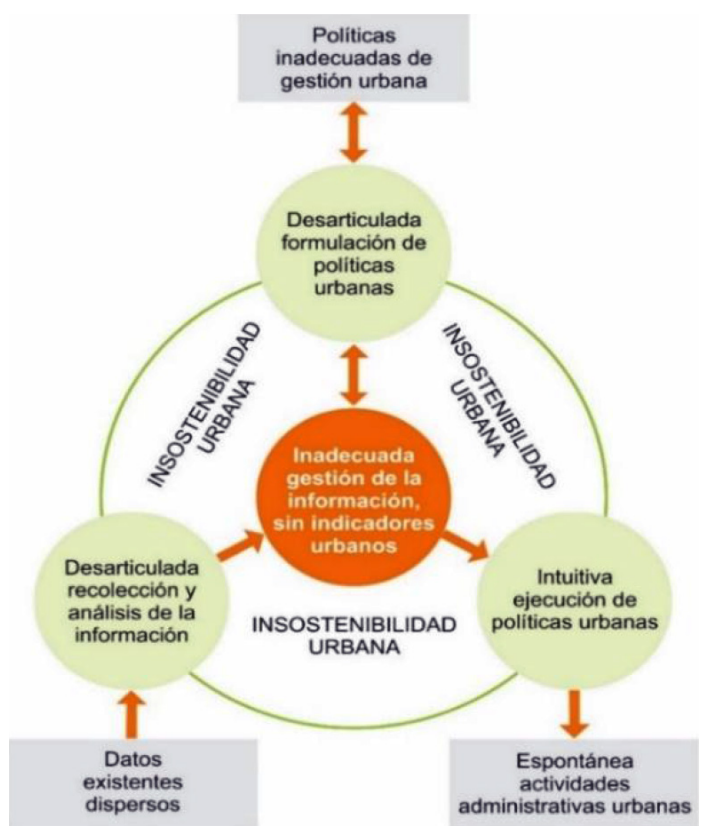

Figura 1. Planteamiento del problema de insostenibilidad urbana. Basado en [5], y adecuación propia.

La Figura 1 retrata la situación de la gestión del medio ambiente en el mundo para el año 1997, se hace latente en nuestro medio al mantenerse la situación descrita. Datos existentes dispersos, que originan una desarticulada recolección y análisis de la información, y ésta a la vez permite una inadecuada gestión de la información urbana que produce dos grandes efectos como son la espontánea actividades administrativas urbanas y la desarticulada formulación de políticas urbanas. De ahí se deduce la pregunta de la formulación del problema en estudio. Al aplicar los indicadores e índices urbanos se logró el objetivo principal determinar cómo influyen los indicadores urbanos en el desarrollo sostenible urbano de Huancayo metropolitano - Perú.

Considera los procesos seguidos en la investigación para alcanzar este objetivo, tal como el análisis de los planes de desarrollo urbano donde no se validó indicador alguno, y estos han sido construidos de manera rigurosa sobre la base de datos de los censos nacionales corroborados por el estudio cartográfico ambiental.

\section{MARCO TEÓRICO}

El marco teórico desarrollado como una etapa subsiguiente al problema y objetivos del estudio, permitió la revisión de los antecedentes, las bases teóricas y la definición conceptual de términos. Barnett \& Parnell [6] proponen, en su trabajo de investigación, la importancia de las ciudades y del desarrollo urbano sostenible y la necesidad de su institucionalización, post agenda 2015. Considera relevante la defensa el activismo y los modos de monitorear y evaluar los procesos de transformación urbana con los indicadores urbanos.

Kitchin [7], en su trabajo de investigación; evalúa las iniciativas, de proyectos de indicadores desarrollados y adoptados por las ciudades, indicando que existe la posibilidad de manipulación de intereses creados que alteran las cuestiones metodológicas y técnicas todavía no reconocidas. Esto evidencia en la investigación su necesidad urgente de aplicación y superar la racionalidad instrumental ingenua.

Turcu [8] a través de su trabajo de investigación; reflexiona sobre la medición de la sostenibilidad, sus métodos y enfoques e incluye el mismo proceso seguido por la investigación, para concluir que los indicadores son una herramienta de medición más influyente de todos. Y concluye con el potencial de su aplicabilidad en los gobiernos locales como es nuestro caso.

El marco teórico permitió confeccionar el diseño metodológico de la investigación, es decir, el momento en que establecimos la información recogida, la analizamos y esta nos proporcionó un conocimiento profundo de la teoría que le da significado a la investigación.

La guía de aplicación: Sistema Integrado de Indicadores Urbanos, Observatorio de Medio Ambiente Urbano (OMAU) elaborado por UNHABITAT/ROLAC [9] es el sistema en que me he basado para el presente estudio debido a que se encuentra vigente y en plena implementación y perfeccionamiento. En este sistema integrado participan las ciudades socias: Calvia (España), 
Málaga (España), Provincia de Treviso (Italia), Rosario (Argentina), Montevideo (Uruguay), Belo Horizonte (Brasil), Corporación Autónoma Regional del Atlántico (Colombia), y Viña del Mar (Chile), y el conjunto de indicadores e índices está representado en la Figura 2.

La Figura 2 presenta 4 dimensiones principales dentro de los cuales se organizan los indicadores urbanos del sistema, según el Observatorio de Medio Ambiente Urbano (OMAU), que servirá de información para la evaluación del desarrollo sostenible urbano de Huancayo metropolitano.

Se adopta la teoría del desarrollo de Manher \& Bunge [10] como marco general que contiene el desarrollo sostenible y sus indicadores del proyecto, de la cual sostenemos que un periodo (o etapa) en la historia de la vida de un organismo es un proceso de desarrollo solo si va acompañado de la emergencia o submergencia de al menos una propiedad (o cualidad) genérica, ya sea composicional o estructural. ...Es más, estipulamos que el cambio cualitativo en cuestión debe ser un evento o proceso interno, es decir, uno que involucre alguna actividad o función organísmica.

Represente $\mathrm{P}(\mathrm{b}, \mathrm{t})$ el conjunto de propiedades genéricas de un biosistema $b$ en algún tiempo $t$. Además, llamemos s al estado b en el tiempo t y s' a su estado en un tiempo t', donde t' $>$ t. Entonces

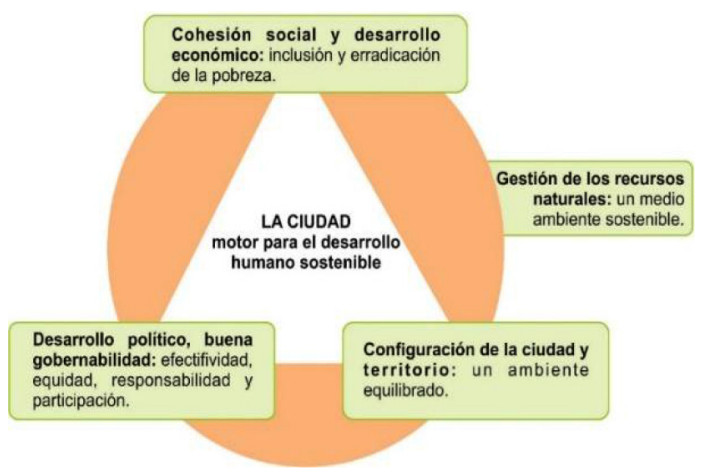

Figura 2. Dimensiones del desarrollo sostenible, OMAU. Basado en [9]. Guía de Aplicación: Sistema Integrado de Indicadores Urbanos, Observatorio de Medio Ambiente Urbano (OMAU), p. 6. Ayuntamiento de Málaga. el evento (o proceso) 〈s, s'> es un evento (o proceso) de desarrollo de b, si y solo si:

(i) 〈s, s'〉 no es (directamente) generado por algún agente $\mathrm{o}$ agentes ambientales; $\mathrm{y}$.

(ii) $\mathrm{P}\left(\mathrm{b}, \mathrm{t}^{\prime}\right) \neq \mathrm{P}(\mathrm{b}, \mathrm{t})$.

Tomamos la ciudad de Huancayo como un biosistema que en un proceso de 48 años (tomado desde el censo de 1961 ), ha cambiado sus propiedades cualitativas genéricas en su estructura y forma.

Utilizando el concepto de sostenibilidad dado por Gallopin [11] en su aplicación al tema urbano podemos definirlo en términos elementales como:

$$
\mathrm{V}\left(S U_{t+1}\right) \geq \mathrm{V}\left(S U_{t}\right)
$$

Donde $\mathrm{V}$ es la función de valuación del estado o condición $(S U)$ del sistema urbano (esto es, un sistema es sostenible cuando el "valor" neto del sistema o de su producto no necesariamente en términos económicos no disminuye en el tiempo t).

\section{METODOLOGÍA}

Son tres metodologías la primera, de cartografía ambiental y su proceso de evolución al estado actual del territorio y la población; la segunda, es la contrastación de indicadores urbanos manejados por los planes urbanos y su posibilidad de utilización; y, la tercera, es la creación de indicadores e índices urbanos para la aplicación del biograma

En la primera metodología de cartografía ambiental se ha tomado como referencia metodológica a McHarg [12], en base a los datos de las fuentes oficiales del Instituto Nacional de Estadística e Informática, el Servicio Aerofotográfica Nacional (SAN), el Instituto Geográfica Nacional (IGN), y OIKONOS empresa privada para las imágenes satelitales. Procesados con sistemas CAD-SIG. Con el objeto de tener una comprobación rápida y panorámica de la situación territorial donde se asienta Huancayo Metropolitano - Perú, producto de ello se tiene el mapa 1 Evolución histórica de Huancayo Metropolitano - Perú 19542016, y el cálculo de densidades en los diversos periodos analizados.

En la segunda metodología, contrastación de indicadores urbanos en los planes urbanos de 
Huancayo Metropolitano, a través de las memorias descriptivas, para ello se ha efectuado la búsqueda de archivos en las instancias locales, regionales, nacionales y privados Se obtuvo los indicadores manejados en los planes urbanos de Huancayo Metropolitano: con la utilización de la memoria explicativa del Ing. Oswaldo Raez Patiño y los siguientes 5 planes urbanos:

- Plan Regulador de Huancayo 1960 realizado por la Oficina Nacional de Planificación y Urbanismo, ONPU [13].

- Plan Director de la Ciudad de Huancayo 1976-1985 realizado por el Consejo Provincial de Huancayo \& Ministerio de Vivienda y Construcción [14].

- Plan Director de la Ciudad de Huancayo 1991 realizado por la Municipalidad Provincial de Huancayo [15].

- Plan Director de la Ciudad de Huancayo 19962005, realizado por la Municipalidad Provincial de Huancayo \& Instituto Nacional de Desarrollo Urbano, INADUR [16].
- Plan de Desarrollo Urbano de la Ciudad de Huancayo 2006-2011, realizado por la Municipalidad Provincial de Huancayo [17], en vigencia actual.

- Plan de Desarrollo Metropolitano 2017-2037 realizado por la Municipalidad Provincial de Huancayo [18], propuesta en consulta para aprobación definitiva, no incluida en el estudio.

En la Figura 3 se esquematiza como se efectuó la contrastación de los indicadores obtenidos de los planes urbanos de Huancayo Metropolitano, con la relación del sistema de indicadores urbanos [9], mediante la elaboración de fichas metodológicas del Sistema Integrado de Indicadores Urbanos Un-Hábitat.

En la tercera metodología para estimar el nivel de desarrollo sostenible de territorios utilizada para la generación del índice de desarrollo sostenible de territorios, se trabajó con el Biograma desarrollado por Sepúlveda [19] en el Instituto Interamericano de Cooperación para la Agricultura (IICA), tiene dos

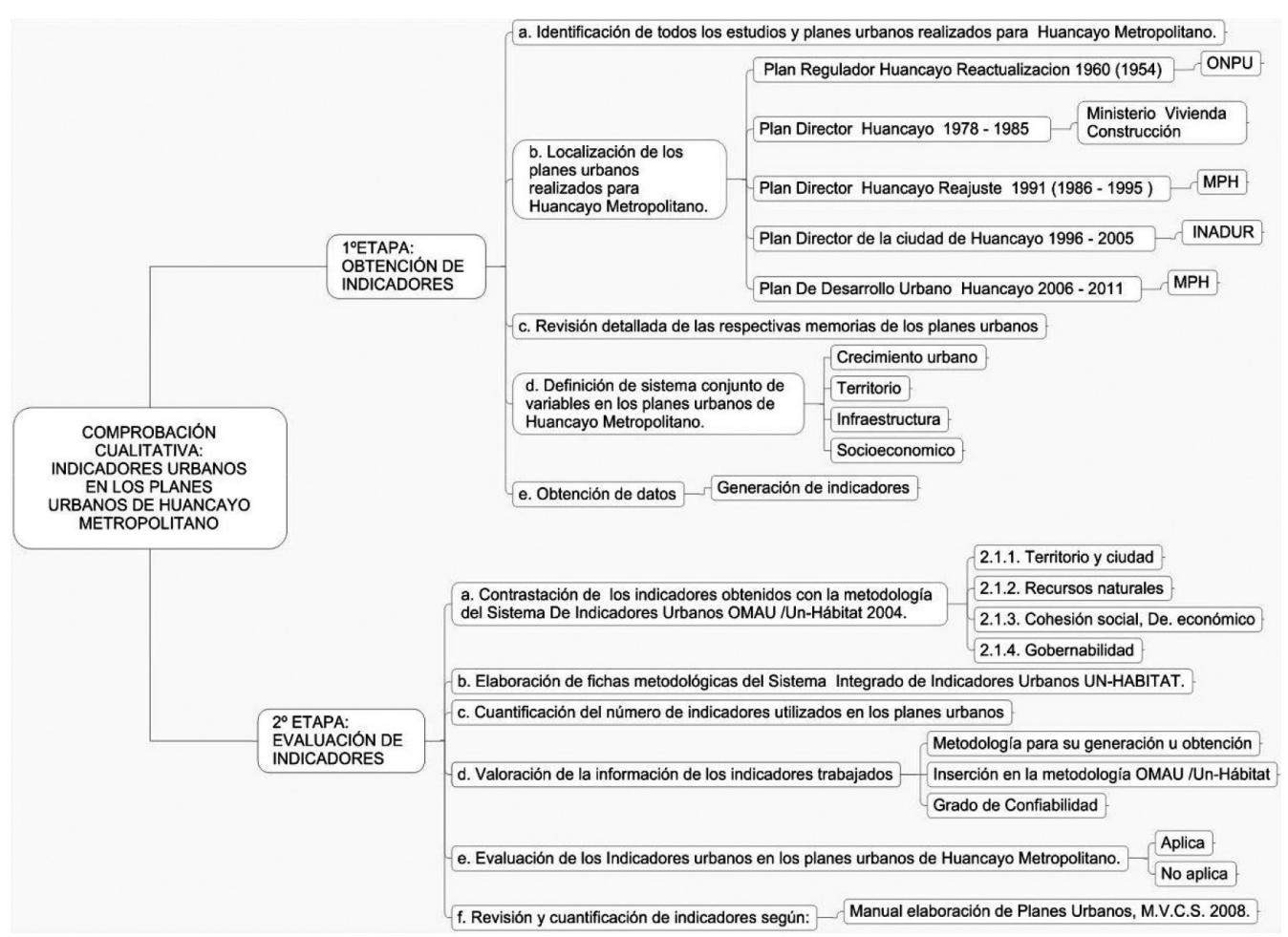

Figura 3. Proceso metodológico: Contrastación de indicadores urbanos en los planes urbanos de Huancayo Metropolitano - Perú. 
referentes importantes: Las fichas metodológicas del sistema integrado de indicadores urbanos del Observatorio de medio Ambiente Urbano (OMAU) de Málaga. Complementado con el PGU - ALC o el CNUAH, denominados Indicadores Urbanos Estambul + 5, el año 2001, y su complemento del 2004.

El Biograma versión final del 2008, ha sido aplicado y corregido periódicamente por más de una década, en temas relacionados al enfoque territorial y se adecua para un ámbito urbano. Para este estudio se fijó cuatro unidades de análisis urbano-municipales, siendo estos los siguientes: Huancayo, Chilca, El Tambo y Huancayo Metropolitano como resumen general de las tres unidades anteriores. En base a periodos de 10 años, 1961, 1971, 1981, 1991, 2001 y la proyección hacia el año 2011, teniendo como referencia de cálculo los censos realizados en 1961 presentado por Oficina Nacional de Estadística y Censos [20], censo de 1972 presentado por Dirección de Censos, Muestreo y Encuestas Especiales [21], censo de 1981 presentado por Instituto Nacional de Estadística [22], censo de 1993 presentado por Instituto Nacional de Estadística e Informática [23], y el último censo efectuado en el Perú del 2007 presentado por Instituto Nacional de Estadística e Informática [24]. Las dimensiones o componentes de los sistemas reflejan de manera integral el estado de las unidades de análisis, para ello se tomaron las dimensiones: Configuración de la ciudad y el territorio, Gestión de los recursos naturales, finalmente Cohesión social y desarrollo económico. En el Biograma se utilizan cinco colores para caracterizar fácilmente el estado de desarrollo sostenible de la unidad de análisis.

En la Tabla 1, se muestra los indicadores, variables y dimensiones utilizados para el análisis de desarrollo sostenible a través el Biograma versión final del 2008.

La población lo constituyen las 8 ciudades cabeceras de macro región excluida Lima e incluida la ciudad de Huancayo, como se muestra en la Figura 4, y la muestra se esquematiza en la Figura 5.

Tabla 1. Indicadores, variables y dimensiones utilizados para el análisis de desarrollo sostenible.

\begin{tabular}{|c|c|c|}
\hline $\begin{array}{c}\text { I. Configuración } \\
\text { de la ciudad y el territorio }\end{array}$ & $\begin{array}{c}\text { II. Gestión } \\
\text { de los recursos naturales }\end{array}$ & $\begin{array}{l}\text { III. Cohesión social y } \\
\text { desarrollo económico }\end{array}$ \\
\hline TC 1.1.0 Crecimiento poblacional & $\begin{array}{r}\text { NR 2.1.0 } \begin{array}{r}\text { Precio de agua, nuevos } \\
\text { soles/100 litros de agua }\end{array} \\
\end{array}$ & \begin{tabular}{|ll} 
SE 3.1.1 & $\begin{array}{l}\text { Tenencia de la vivienda: vivienda } \\
\text { propia }\end{array}$ \\
\end{tabular} \\
\hline TC 1.2.0 Densidad poblacional & $\begin{array}{c}\text { NR 2.2.0 Consumo de energía. } \\
\text { Consumo per cápita. }\end{array}$ & \begin{tabular}{|l} 
SE 3.1.2 \\
$\begin{array}{l}\text { Tenencia de la vivienda: vivienda } \\
\text { alquilada }\end{array}$
\end{tabular} \\
\hline TC 1.3.0 Tamaño medio de un hogar & $\begin{array}{l}\text { NR 2.3.1 } \text { Gestión integral de de- } \\
\text { sechos sólidos: \% de ho- } \\
\text { gares que cuenta con } \\
\text { recolección }\end{array}$ & \begin{tabular}{|ll} 
SE 3.1.3 & Tenencia de la vivienda: vivienda \\
& otros \\
SE 3.2.0 & Acceso a saneamiento \\
SE 3.3.0 & Acceso a agua potable \\
SE 3.4.1 & Conexiones a tecnologías de \\
& información y comunicación: \\
& servicio de telefonía fija \\
SE 3.4.2 & Conexiones a tecnologías de \\
& información y comunicación: \\
& computadoras
\end{tabular} \\
\hline TC 1.4.0 Tasa de formación de hogares & $\begin{array}{l}\text { NR 2.3.2 Gestión integral de } \\
\text { desechos sólidos: volu- } \\
\text { men semanal }\end{array}$ & $\begin{array}{c}\text { SE 3.5.0 Cobertura del servicio de energía } \\
\text { eléctrica }\end{array}$ \\
\hline \multirow[t]{2}{*}{$\begin{array}{l}\text { TC 1.5.0 Población urbana/Población } \\
\text { total }\end{array}$} & $\begin{array}{c}\text { NR 2.3.3 } \text { Gestión integral de dese- } \\
\text { chos sólidos: destino } \\
\text { relleno sanitario }\end{array}$ & SE 3.6.0 Tasa de desempleo totales \\
\hline & & $\begin{array}{|ll|}\text { SE 3.7.1 } & \text { Tasa de alfabetización } \\
\text { SE 3.7.2 } & \begin{array}{l}\text { Brecha de alfabetización según } \\
\text { sexo }\end{array}\end{array}$ \\
\hline
\end{tabular}




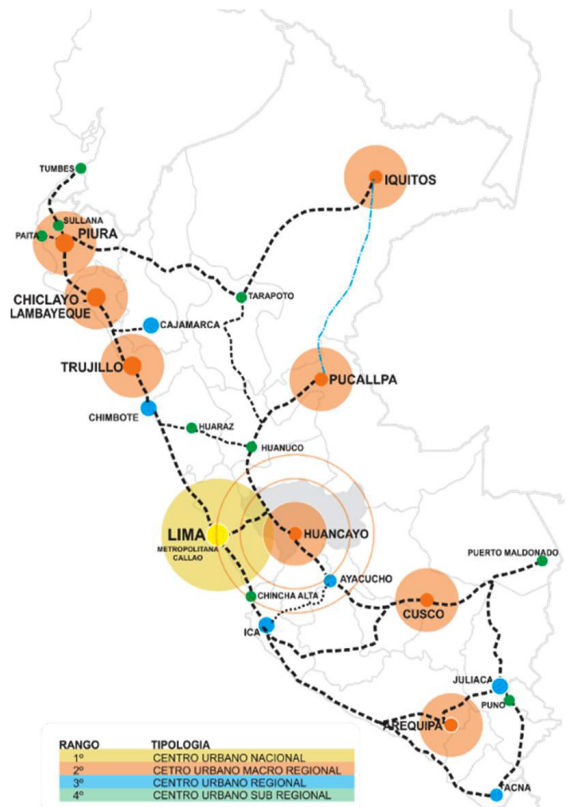

Figura 4. Sistema Nacional de Centros Poblados en el Perú: Sistemas y Subsistemas, basado en el Ministerio de Vivienda Construcción y Saneamiento [4].

\section{RESULTADOS}

\section{Resultados de la cartografía ambiental}

En la Figura 6: Evolución histórica de Huancayo Metropolitano - Perú 1954-2016, y Figura 7: Densidad urbana para análisis de sostenibilidad periódica- Biograma 1961, 1971, 1981, 1991, 2001 y 2011, muestra el crecimiento tanto del área urbana, como de la población de Huancayo Metropolitano, y el decrecimiento en la densidad poblacional de acuerdo a las siguientes características obtenidas de la proyección de datos, en el año 1961 se tenía una superficie de 553 has, población de 64153 habitantes, y densidad de 115 hab/ha, hasta el año 2011 con una superficie de 3676 has, 344410 habitantes y densidad de 93 hab/ha, resultando que entre los años mencionados la superficie, creció 6 veces, la población 5,36 veces, y la densidad decreció a 0,8 veces.

Así mismo, el área urbana de El Tambo supera al de Huancayo y Chilca.

A diferencia de la población y superficie urbana, los cuales tienen un incremento sostenido en el tiempo, el

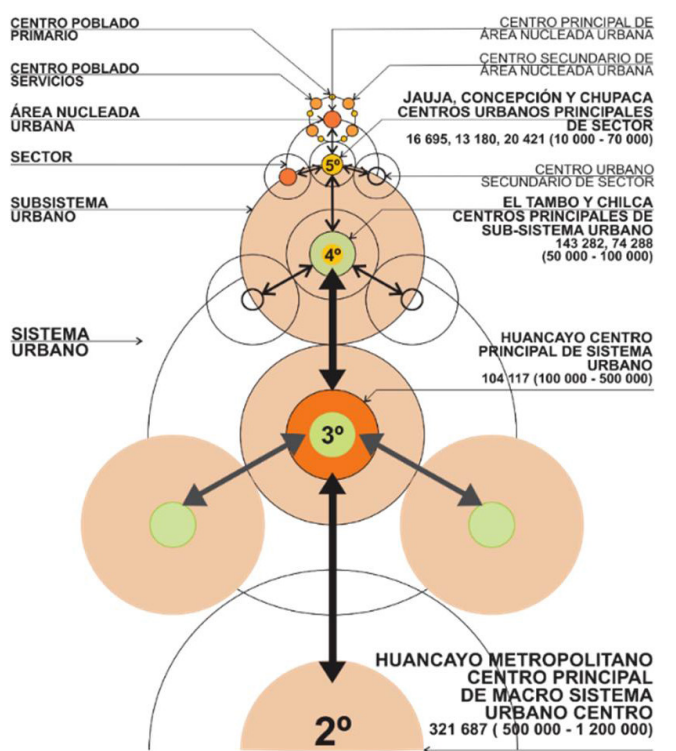

Figura 5. La muestra en el marco del Macrosistema Centro del Perú. Adaptada del Sistema Nacional de Centros Poblados: Macrosistema Centro elaborada por el Ministerio de Vivienda Construcción y Saneamiento [4].

aspecto de densidad poblacional viene disminuyendo. En término metropolitano existe la tendencia a una ciudad extendida, observando contradicción con las reglamentaciones sobre densidad urbana de los diferentes Planes de Desarrollo Urbano, incluido el actual proyectado del 2006 al 2011.

El sistema de indicadores es producto de la revisión de:

a) Memorias descriptivas de los planes urbanos de la ciudad de Huancayo.

b) Del manual para la elaboración de planes de desarrollo urbano, 2008 del Ministerio Vivienda, Construcción y Saneamiento, INICAM.

c) Del sistema de Indicadores Urbanos OMAU - UN-HÁBITAT- Programa de las Naciones Unidas para Asentamientos Humanos.

En las Figuras 8 y 9, se presenta los resultados de la contrastación de la Tabla 2, quedando calificados 14 indicadores de los planes urbanos, y al ser sometidos a la lista de cotejo de verificación metodológica, solo 1 indicador aplica; los 13 indicadores, restantes presentan información con fallas metodológicas en su obtención, de acuerdo a la metodología UNHÁBITAT y consignan datos no confiables, por lo que es necesario construir sobre la base censal 


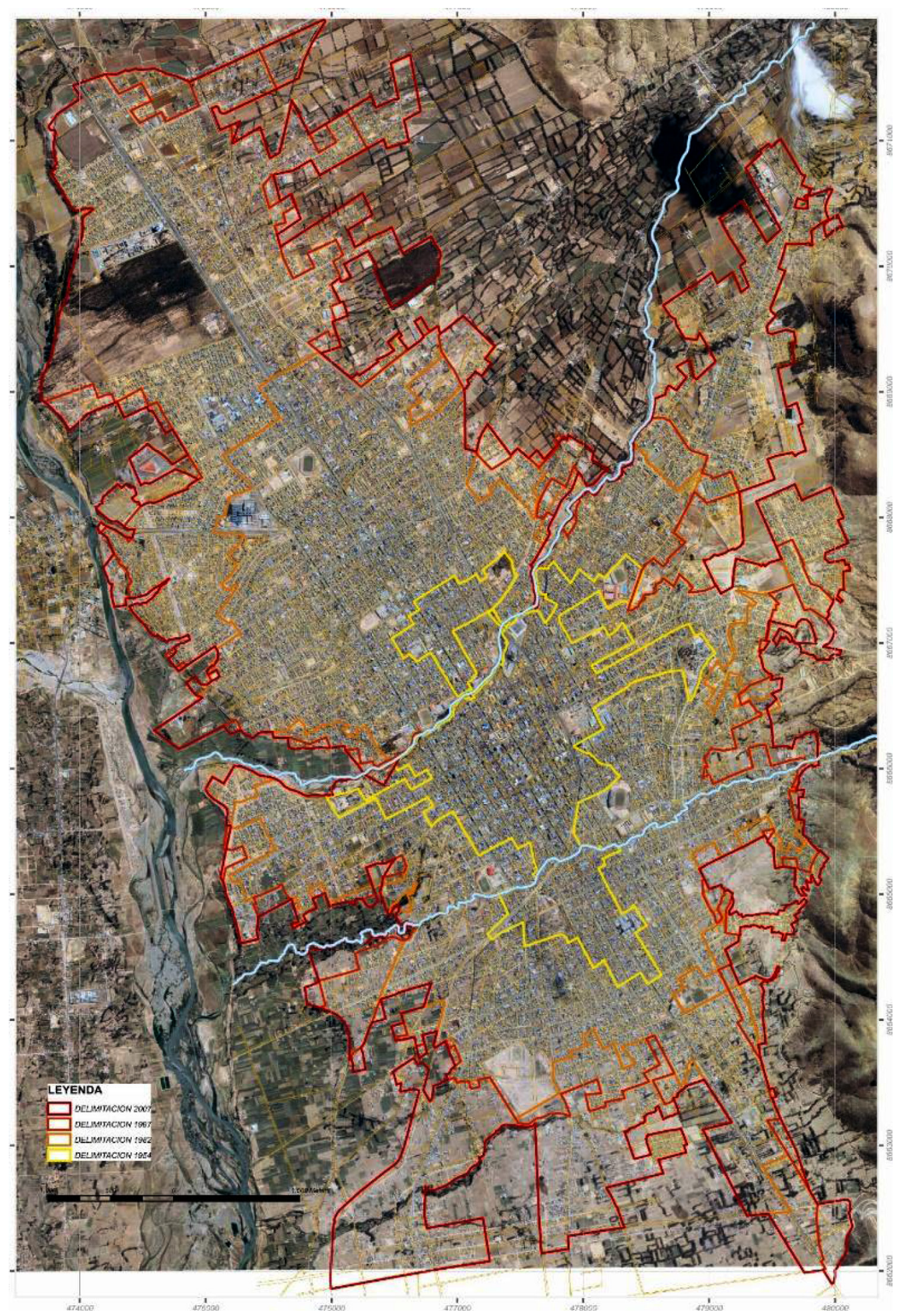

Figura 6. Evolución histórica de Huancayo Metropolitano - Perú 1954-2016, Imagen satelital y aerofotografía SAN, se delimitó perímetros urbanos en base a los perímetros establecidos en planes urbanos correspondientes. 


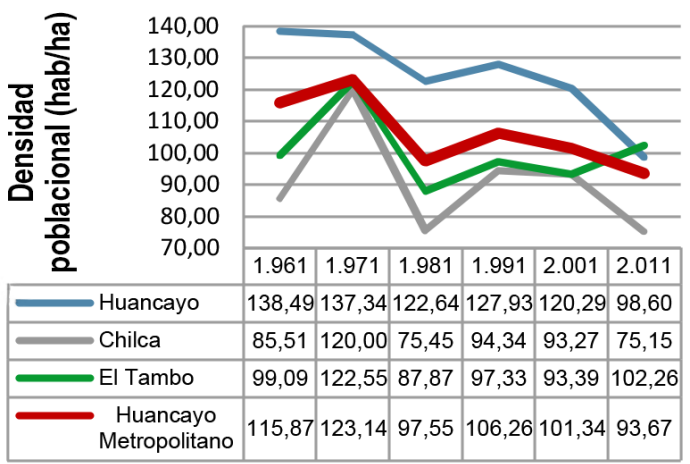

Figura 7. Densidad urbana para análisis de sostenibilidad periódica - Biograma 1961, 1971, 1981, 1991, 2001 Y 2011 Censos nacionales y aerofotografía. Resultados de contrastación de indicadores urbanos en los planes urbanos de Huancayo Metropolitano.

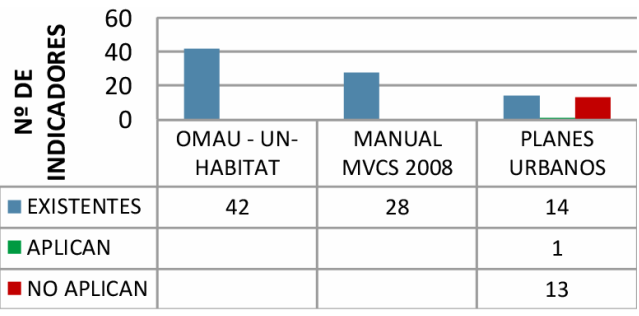

Figura 8. Evaluación de los indicadores utilizados en los planes urbanos de Huancayo M.

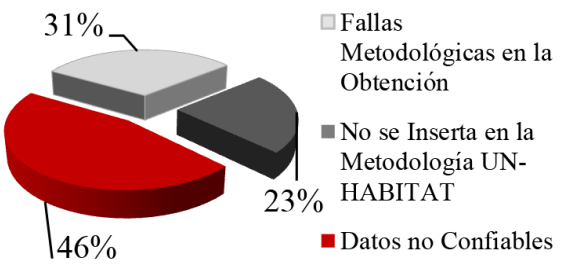

Figura 9. Causas por las que los indicadores procesados no aplicaron.

Tabla 2. Contrastación de indicadores de los planes urbanos, el manual del MVCS y OMAU-2006, UN-HÁBITAT 2004.

\begin{tabular}{|c|c|c|c|c|c|}
\hline \multicolumn{2}{|c|}{$\begin{array}{c}\text { Planes Hurbanos - Huancayo } \\
\text { (a) }\end{array}$} & \multicolumn{2}{|r|}{$\begin{array}{c}\text { Manuel MVCS } 2018 \\
\text { (b) }\end{array}$} & \multicolumn{2}{|r|}{$\begin{array}{l}\text { OMAU, Un Hábitat } \\
\text { (c) }\end{array}$} \\
\hline \multirow{5}{*}{ 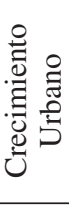 } & Población & \multirow{5}{*}{ 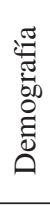 } & Crecimiento Poblacional & \multirow{5}{*}{ 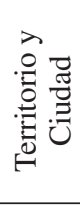 } & Crecimiento Poblacional \\
\hline & Superficie & & Densidad Poblacional & & Densidad Poblacional \\
\hline & Densidad Poblacional & & Población Urbana por sexo & & Usos del suelo \\
\hline & Demanda de área para la & & Distribución Espacial de & & Espacio público \\
\hline & Expansión Urbana & & la Población Urbana & & Transporte \\
\hline \multirow{6}{*}{ 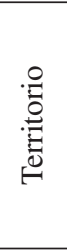 } & \multirow{2}{*}{ Usos del suelo } & \multirow{6}{*}{ 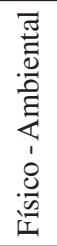 } & Usos del suelo & \multirow{6}{*}{ 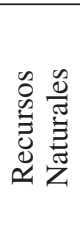 } & Calidad del Aire \\
\hline & & & Vialidad y Transporte & & Calidad del Agua \\
\hline & Características de & & Equipamiento Urbano & & Consumo Per cápita Agua \\
\hline & Edificación & & Servicios Públicos & & Destino Residuos Sólidos \\
\hline & \multirow{2}{*}{$\begin{array}{l}\text { Contaminación en ríos } \\
\text { (breve descripción) }\end{array}$} & & Recursos Naturales & & Cobertura Vegetal \\
\hline & & & Zonas de Riesgo & & Biodiversidad \\
\hline \multirow{7}{*}{ 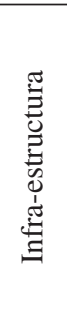 } & \multirow{2}{*}{ Equipamiento urbano } & \multirow{7}{*}{$\begin{array}{l}\frac{\pi}{8} \\
8 \\
\text { n }\end{array}$} & Acceso a servicios básicos & \multirow{7}{*}{ 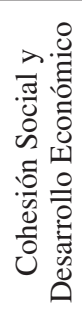 } & Vivienda \\
\hline & & & Pobreza & & Educación \\
\hline & \multirow{3}{*}{ Vialidad y transporte } & & Empleo & & Salud \\
\hline & & & Nivel Educativo & & Servicio \\
\hline & & & Salud & & $\begin{array}{l}\text { Empleo y Desarrollo } \\
\text { Económico }\end{array}$ \\
\hline & \multirow{2}{*}{ De servicios } & & Niveles Socioeconómicos & & Pobreza \\
\hline & & & Desarrollo Humano & & Desarrollo Humano \\
\hline \multirow{5}{*}{ 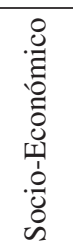 } & Demografía & \multirow{5}{*}{ 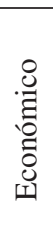 } & Producción por Sectores & \multirow{5}{*}{ 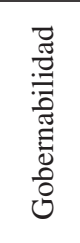 } & $\begin{array}{l}\text { Efectividad } \\
\text { Gubernamental }\end{array}$ \\
\hline & Vivienda & & Actividades Económicas & & Ingresos Gobierno Local \\
\hline & Precariedad Predial & & Informalidad & & Equidad de Género \\
\hline & Actividades Económicas & & Distribución Espacial de & & Participación Ciudadana \\
\hline & Comercio Formal & & Actividades Económicas & & Asociaciones Cívicas \\
\hline
\end{tabular}


los nuevos indicadores para ser analizados en el biograma como lo indica la Figura 10.

Con el fin de adaptar los indicadores a una escala común, se utilizó una función de relativización, la cual se basa en la metodología planteada por el PNUD para calcular el índice de desarrollo humano. Para el caso en que los indicadores presentan una relación positiva se adoptó la siguiente ecuación (1):

$$
F(x)=\frac{x=m}{M-m}
$$

Para el caso en que los indicadores presentan una relación inversa, se modificó la fórmula anterior con el fin de mantener sus propiedades:

$$
f(x)=\frac{x-m}{m-M}
$$

En tales fórmulas:

$\mathrm{x}=$ es el valor correspondiente de la variable o indicador para una unidad de análisis determinada en un período determinado.

$\mathrm{m}=$ es el valor mínimo de la variable en un período determinado.

$\mathrm{M}=$ es el nivel máximo en un período determinado.

Mediante la utilización de estas fórmulas se obtienen índices individuales para cada indicador, los cuales fluctúan entre 0 y 1 . Para ambos casos (cuando los indicadores presentan una relación positiva o negativa), un valor de 1 representa una mejor situación, contrario a un valor de 0 , en cuyo caso representa la peor situación. Las fórmulas anteriores permiten relativizar todos los indicadores y, por ende, generan un nuevo set apto para realizar análisis comparativo.

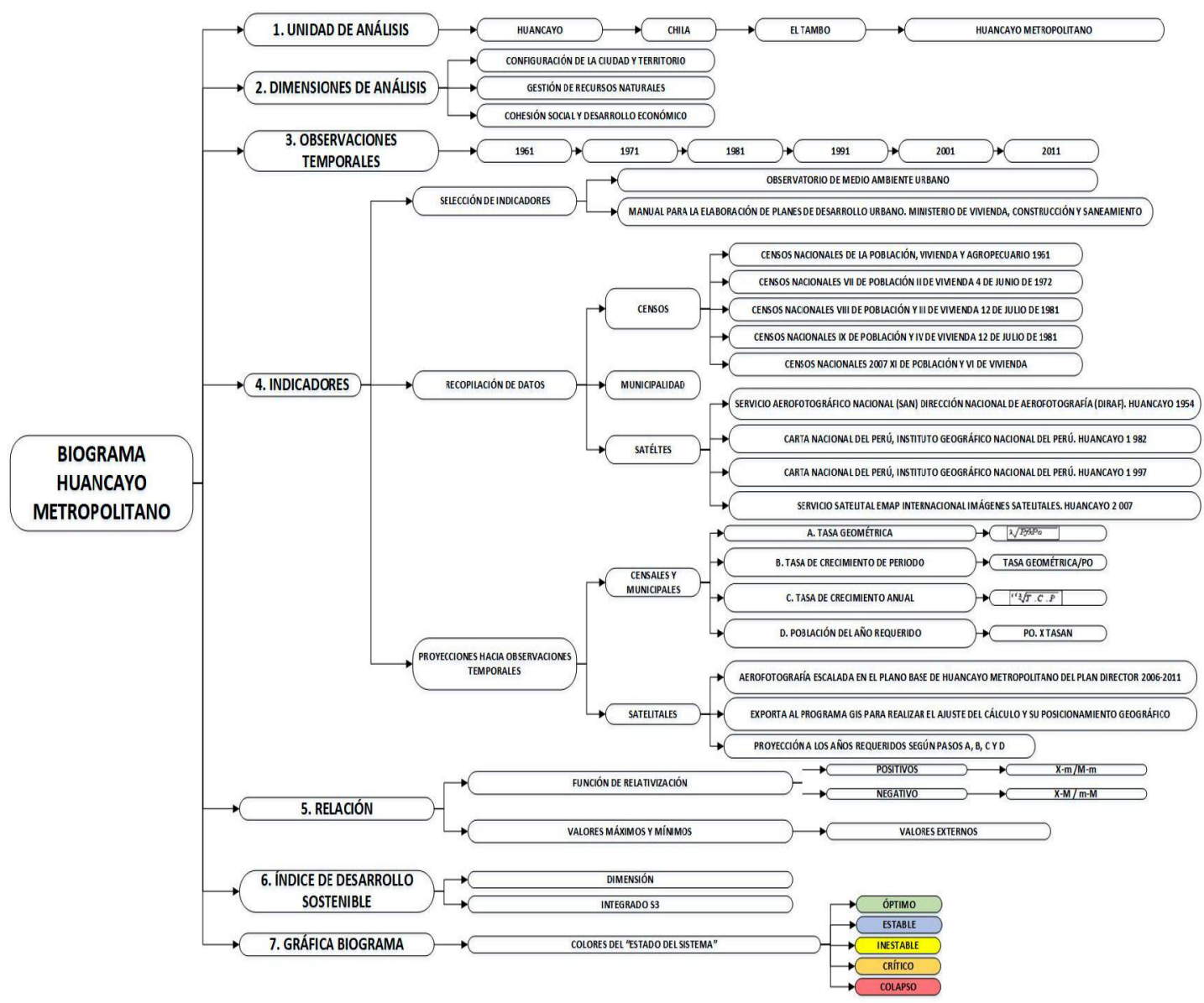

Figura 10. Procedimiento de estimación del nivel de desarrollo sostenible de territorios, Biograma 2008. 


\section{- Niveles máximos y mínimos}

Como se observa en las ecuaciones (1) y (2), con el fin de hacer comparables los indicadores, es necesario establecer un valor máximo y un valor mínimo para cada categoría de los datos que se analizarán. Para tal propósito, la opción más directa es adoptar simplemente el mayor y el menor de los valores observados. Ese procedimiento permite obtener un indicador que refleja una posición relativa con respecto al período de tiempo analizado. De tal manera, el indicador mostrará un valor de 1 en el período en que obtuvo el nivel máximo observado y de 0 cuando se presenta su nivel mínimo.

El procedimiento para hallar valores máximos y mínimos, fue hallado en función a valores observados.

\section{- Valores extremos}

La determinación de los valores extremos se puede realizar mediante un análisis estadístico independiente de las series de datos. De cualquier manera, se establece así un valor límite a partir del cual se considera que surgen esos valores extremos. Así mismo, puede establecer solamente un máximo, permitiendo que el valor mínimo sea el observado, o bien, establecer el nivel mínimo, sin alterar el mayor valor observado como el máximo. Al no considerar este tipo de valores, los datos de la serie se mantendrán dentro de un rango de fluctuación normal. Los límites de fluctuación también son funcionales al trabajar con series de datos pequeñas. En el caso de no contar con series de tiempo extensas que muestren el comportamiento normal de una unidad de estudio, el procedimiento para establecer valores extremos permite tener patrones de comparación para los datos recopilados.

Para el cálculo del índice de desarrollo sostenible, se utiliza una ecuación (ecuación 3) que primero calcula el promedio ponderado de los indicadores de cada una de las dimensiones, previamente relativizados. La fórmula para calcular el Índice de cada dimensión es la siguiente:

$$
S_{D}=\frac{1}{n_{D}} \sum_{i=1}^{n_{D}}{ }_{i}{ }_{i}^{D}
$$

En donde es el indicador de la dimensión $\mathrm{D}$ y se entiende que esa dimensión tiene indicadores. Por tanto es un promedio de los indicadores de la dimensión, los cuales han sido previamente estandarizados, para que tomen valores entre 0 y 1 .

Luego los índices de todas las dimensiones se agregan para obtener el índice integrado. La agregación se hace ponderando cada dimensión por un porcentaje de importancia $\left(\beta_{D}\right)$ La ecuación para calcular el índice integrado de desarrollo sostenible es (ecuación 4):

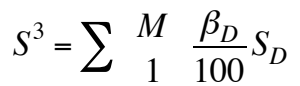

Los porcentajes de importancia determinados para Huancayo Metropolitano fueron los siguientes: $\beta$ para configuración de la ciudad y el territorio es $0,35, \beta$ para Gestión de los recursos naturales es 0,30 y $\beta$ para cohesión social y desarrollo económico.

Resultados del Área urbana de Huancayo metropolitano

En la Figura 11 se presenta el Índice Integrado de desarrollo sostenible por decenios 1961-2011 de Huancayo Metropolitano, cuyos resultados muestran:

1961, sistema crítico. $(0,37)$

1971 , sistema crítico. $(0,30)$, menor crecimiento

1981, sistema crítico. $(0,32)$

1991, sistema inestable. $(0,40)$

2001, sistema inestable. $(0,50)$

2011, sistema estable. $(0,69)$, mayor crecimiento.

Logros de la investigación:

- Efectuar el diagnóstico de los planes urbanos, y sistemas de gestión urbana, para el caso de Huancayo Metropolitano, como situación que pronostica la situación de las ciudades en el contexto nacional.

- Obtener la primera valoración del desarrollo sostenible en el área urbana de Huancayo Metropolitano

- En el programa Hábitat de las Naciones Unidas se tiene el programa del observatorio urbano, en el cual están inscritas 353 ciudades del mundo y desarrollando los indicadores urbanos, de América Latina y el Caribe se tienen 39 ciudades lideradas por Brasil con 15 ciudades, secundada por México con 7 ciudades, del Perú no tiene inscrita ninguna ciudad. De ahí la importancia en crear la necesidad del uso de los indicadores urbanos, como un instrumento de gestión urbana. 

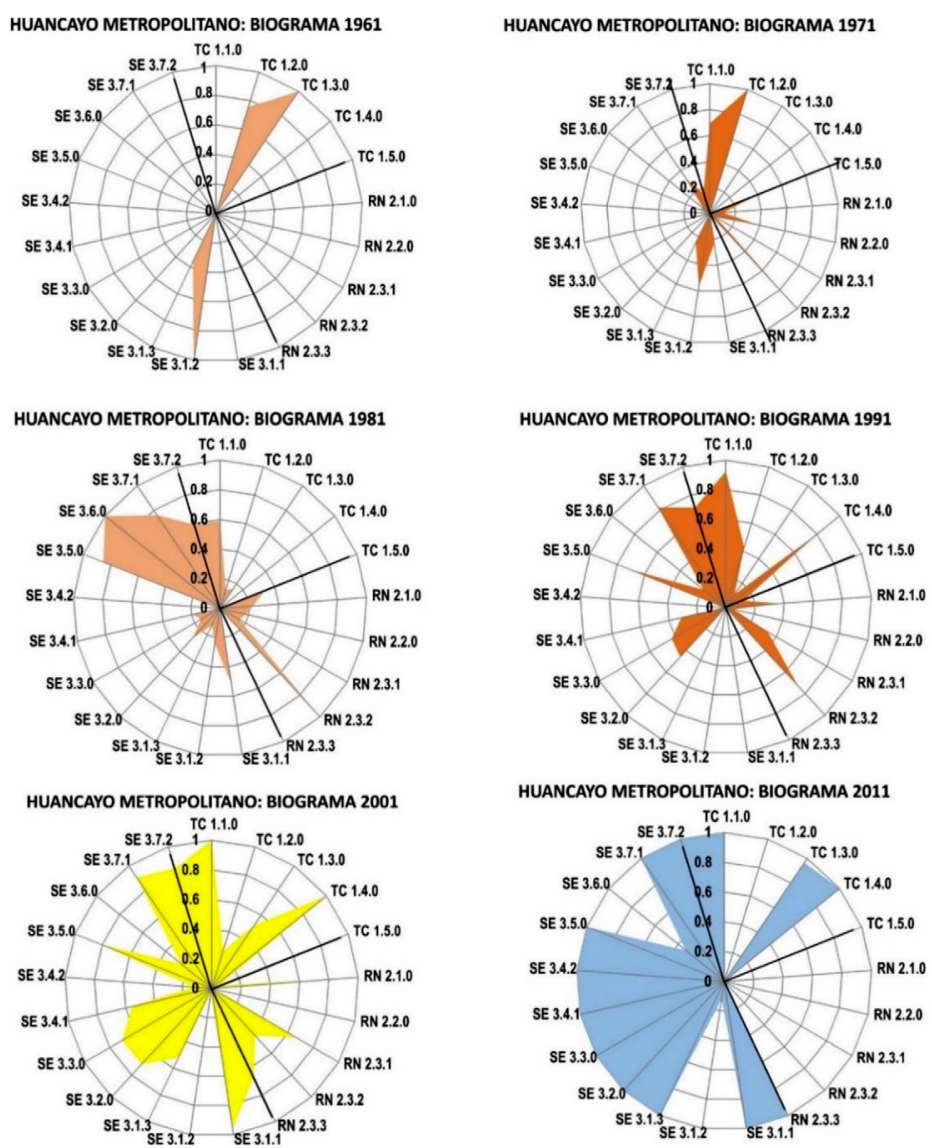

Figura 11. Resumen de tendencias de índices integrados de desarrollo sostenible $S^{3}$ para de Huancayo Metropolitano, en el horizonte de 50 años, con periodos de cada 10 años. En base a la aplicación del método de Biograma 2008.

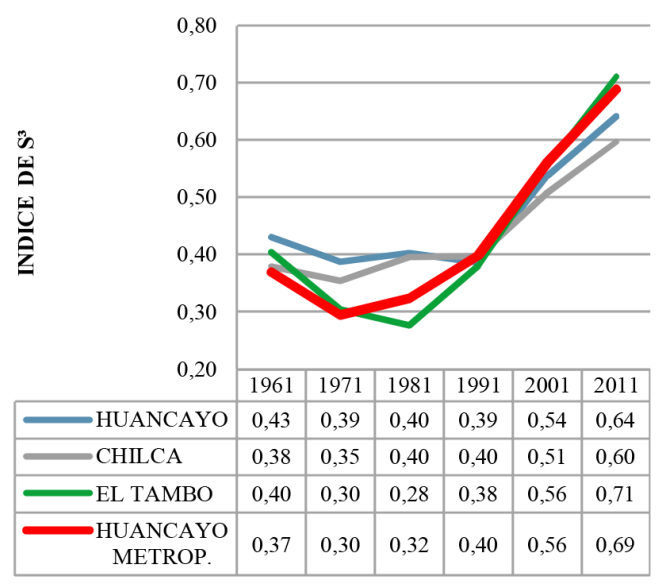

Figura 12. Muestra del área urbana del distrito de El Tambo, está en franco progreso en el horizonte de 50 años evaluados

\section{DISCUSIÓN Y CONCLUSIÓN}

Del resultado obtenido y como muestra la Figura 13, se cumple con la teoría del desarrollo sostenible adoptado, Huancayo Metropolitano como un biosistema que en un proceso de 48 años (tomado desde el censo de 1961), ha cambiado sus propiedades cualitativas genéricas en su estructura y forma. Además, llamemos s a su estado según el índice de desarrollo 0,37 (b) en el año 1961(t) y s' a su estado según el índice de desarrollo 0,69 (b) en el año $2011(\mathrm{t}$ '), donde $\mathrm{t}$ '>t. Entonces el proceso $<\mathrm{s}$, s'> es un proceso de desarrollo de b.

El sistema integrado del OMAU se constituye de cuatro dimensiones, las tres primeras fueron trabajadas de acuerdo a la metodología, quedando 
pendiente la dimensión de gobernabilidad por carecer de información en el horizonte de estudio. Respecto a cada dimensión esta tiene los siguientes resultados; la dimensión de configuración de la ciudad y el territorio en general es de tendencia a la inestabilidad, sostenida por el área urbana del El Tambo que eleva los índices sin llegar a la estabilidad, debido a que Huancayo y Chilca tienden caer sostenidamente. La dimensión de gestión de recursos naturales en general es de tendencia a la estabilidad, teniendo el mismo comportamiento Huancayo, Chilca y El Tambo. Y la dimensión cohesión social y desarrollo económico en general tiende a ser óptima, los resultados muestran crecimiento en todos los componentes de Huancayo Metropolitano.

En la Regresión y Correlación de la muestra se afirma con un $95 \%$ de confianza, que los indicadores urbanos en su conjunto influyen en el desarrollo sostenible urbano de Huancayo metropolitano. La Contrastación de Hipótesis específicas utilizó el Modelo de Correlación de Pearson, para determinar la significancia de cada una de las dimensiones y de la variable dependiente desarrollo sostenible urbano. Los indicadores urbanos de gestión de los recursos naturales influyen en el desarrollo sostenible urbano de Huancayo metropolitano, Los indicadores urbanos de cohesión social y desarrollo económico también influyen en el desarrollo sostenible urbano de Huancayo metropolitano, a diferencia de los indicadores urbanos de configuración de la ciudad y el territorio que no influyen en el desarrollo sostenible urbano de Huancayo metropolitano. Reflejo de la no influencia de la dimensión de configuración de la ciudad y el territorio, en la que se incluyen los indicadores de espacio público, se percibe la calidad y la cantidad de los espacios públicos en Huancayo metropolitano, los cuales no cumplen con los estándares mínimos establecidos.

Se considera que prácticamente no existe indicador valido en los planes urbanos 1960-2011, así como se evidencia la dimensión configuración de la ciudad y el territorio que es el índice propicio para evaluar la aplicación de planes urbanos, producto de ello se establece la necesidad inmediata de operar la ciudad con indicadores urbanos de manera que pueda monitorearse el desarrollo sostenible de la misma y así la ciudad sostenible no se reduzca a un simple discurso político.

\section{RECOMENDACIONES}

$\mathrm{Al}$ sistema nacional liderado por el ministerio de vivienda, incluirse de manera urgente al observatorio urbano de Latinoamérica, o en su defecto implementar su propio sistema a través del observatorio urbano con indicadores urbanos a nivel nacional, regional y local. Para la estimación del desarrollo sostenible de territorios urbanos, que permita desarrollar y mejorar la gestión urbana de manera permanente, en cada uno de los aspectos urbanos.

\section{REFERENCIAS}

[1] C. Martínez. "Vulnerabilidad ambiental en el valle del Mantaro por las actividades urbanas". Colegio de Huancayo, Perú: CFMV. 2006.

[2] C. y S. Ministerio de Vivienda. "Sistema Nacional de estandares de urbanismo". Lima, Perú. 2011.

[3] Conferencia de las Naciones Unidas sobre el Medio Ambiente y el Desarrollo. "Informe de la conferencia de las Naciones Unidas sobre el Medio Ambiente y el Desarrollo en Rio de Janeiro". Nueva york. 1992.

[4] Ministerio de Vivienda Construcción y Saneamiento. Reglamento de Acondicionamiento Territorial y Desarrollo Urbano No 022-2016-Vivienda. Lima, Perú. pp. 45. 2016.

[5] L. Velásquez. "Propuesta de Una Metodología de Planificación para el Desarrollo Urbano Sostenible y Diseño de un Sistema de Evaluación de la Sostenibilidad de las ciudades Medianas de América Latina”. Tesis Doctoral en Planificación Urbana. Universidad Politécnica de Catalunya, Barcelona, España. 2003.

[6] C. Barnett and S. Parnell. "Ideas, implementation and indicators: epistemologies of the post-2015 urban agenda". Environ. Urban. Vol. 28 N $^{\circ}$ 1, pp. 87-98. 2016.

[7] R. Kitchin, T.P. Lauriault and G. McArdle. "Knowing and governing cities through urban indicators, city benchmarking and real-time dashboards". Reg. Stud. Reg. Sci. Vol. $2 \mathrm{~N}^{\circ}$ 1, pp. 6-28. 2015.

[8] C. Turcu. "Re-thinking sustainability indicators: local perspectives of urban 
sustainability”. J. Environ. Plan. Manag. Vol. $56 \mathrm{~N}^{\circ}$ 5, pp. 695-719. 2013.

[9] UN-HABITAT/ROLAC. "Guia de Aplicación: Sistema Integrado de Indicadores Urbanos, Observatorio de Medio Ambiente Urbano (OMAU), Ayuntamiento de Málaga”. 2006.

[10] M. Manher and M. Bunge. Fundamentos de Biofilosofía. México: Siglo XXI editores. 2000.

[11] G. Gallopin. "Los indicadores de desarrollo sostenible: Aspectos conceptuales y metodológicos", in Seminario de expertos sobre indicadores de sostenibilidad. 2006. pp. 36.

[12] I. McHarg. Proyectar con la Naturaleza. Barcelona, España: Gustavo Gili. 2000.

[13] Oficina Nacional de Planificación y Urbanismo. "Reactualización Plan Regulador de Huancayo 1954-1980”. Autor. Huancayo, Perú. 1960.

[14] Consejo Provincial de Huancayo y Ministerio de Vivienda y Construcción. "Plan director de Huancayo 1976-1985”. Huancayo. 1976.

[15] Municipalidad Provincial de Huancayo. "Reajuste del Plan Director de la Ciudad de Huancayo 1986-1995”. Autor, Huancayo, Perú, 1991.

[16] Municipalidad Provincial de Huancayo e Instituto Nacional de Desarrollo Urbano. "Plan Director De Huancayo 1996-2005". Autor. Huancayo, Perú. 1996.

[17] Municipalidad Provincial de Huancayo. Plan de Desarrollo Urbano 2006-2011.
Huancayo, Perú: OM No 450-CM/MPH. 2006, pp. 316.

[18] Municipalidad Provincial de Huancayo. Plan de Desarrollo Metropolita $N^{\circ}$ Huancayo. Perú. 2016.

[19] S. Sepúlveda S. "Metodología para estimar el nivel de desarrollo sostenible de territorios", El Biogram. San José, Costa Rica: IICA. 2008.

[20] Oficina Nacional de Estadística y Censos. "Censos Nacionales Poblacion, Vivienda y Agropecuario 1961 - (Volumen XI: Departamento de Junín)". Autor. Lima, Perú. 1970.

[21] Dirección de Censos, Muestreo y Encuestas Especiales. "Censos nacionales VII de población II de vivienda 4 de Junio de 1972: Departamento de Junin", Lima: Oficina Nacional de Estadística y Censos (ONEC). 1975.

[22] Instituto Nacional de Estadística. "Censos Nacionales VIII de Población y III de Vivienda". Direcciones Generales de Censos y Encuestas. Lima, Perú. 1984.

[23] Instituto Nacional de Estadística e Informática (INEI). "Sistema de recuperación de Datos Censos nacionales IX de Población y IV de Vivienda 1993. Censo de Población y vivienda". 1993.

[24] Instituto Nacional de Estadística e Informática (INEI). "Censos nacionales 2007 XI de Población y VI de Vivienda. Sistema de consulta de Datos de Centros Poblados (CCPP) y población Dispersa”. Junín. 2007. 